\author{
From: CELLULAR MECHANISMS OF CONDITIONING \\ AND BEHAVIORAL PLASTICITY \\ Edited by Charles D. Woody, Daniel L. Alkon, \\ and James L. McGaugh \\ (Plenum Publishing Corporation, 1988)
}

$\vdots$

\title{
Electrodiffusion Model of Electrical Conduction in Neuronal Processes
}

\author{
NING QIAN AND TERRENCE J. SEJNOWSKI
}

\section{INTRODUCTION}

The cable model of electrical conduction in neurons is central to our understanding of information processing in neurons. The conduction of action potentials in axons has been modeled as a nonlinear excitable cable (Hodgkin and Huxley, 1952), and the integration of postsynaptic signals in dendrites has been studied with analytic solutions to passive cables (Rall, 1977). Recently, several groups have examined the possibility of more complex signal processing in dendrites with complex morphologies and excitable membranes by numerical integration of the cable equations (Shepherd et al., 1985; Koch et al., 1983; Rall and Segev, 1985; Perkel and Perkel, 1985).

The cable equation is based on an electrical conductance model in which driving forces arising from ionic concentration differences across the membrane are represented by batteries in series with conductances. This model can be derived as an approximation to the Nernst-Planck equation for electrodiffusion. In this chapter we introduce an electrodiffusion model of electrical conduction in one dimension-along the longitudinal dimension of a thin process. In this preliminary report we determine conditions under which the electrical conductance model may not be valid. Complications such as cytoplasmic cisternae, membrane pumps, and ionic buffers will be considered in a later paper.

\section{LIMITATIONS OF THE ELECTRICAL CONDUCTANCE MODEL}

The membrane battery potentials in the electrical conductance model are usually obtained from the Nernst equation and are considered constants. This is a good approximation in the squid giant axon and large neurons but may introduce errors if the concentrations of some ions change significantly. This is more likely to occur in small processes and during synaptic events in small structures such as spines (Rall, 1978; Koch and Poggio, 1983).

A second limitation of the electrical conductance model is in the treatment of lon-

NING QIAN and TERRENCE J. SEJNOWSKI • Department of Biophysics, Johns Hopkins University, Baltimore, Maryland 21218. 
gitudinal current spread within neurons. Only the potential gradient in the cytoplasm is considered, and not concentration gradients. This is usually a good assumption, but the concentration gradients can be large when spatial compartments are small and for some ions like $\mathrm{Ca}^{2+}$, whose concentration can change dramatically under some circumstances.

One additional observation is that different ions may have different concentrationdependent cytoplasmic resistivities, but in the electrical conductance model only the total cytoplasmic resistivity is usually considered.

In the following sections we first derive a set of equations that govern the electrodiffusion of ions in thin cables and then present numerical solutions to these equations for an excitatory postsynaptic potential on a dendritic spine.

\section{ELECTRODIFFUSION MODEL}

The movement of ions in neurons is governed by the Nernst-Planck equation (Jack et al., 1975):

$$
\bar{J}_{i}=-D_{i}\left[\bar{\nabla} n_{i}+\left(n_{i} / \alpha_{i}\right) \bar{\nabla} V\right]
$$

where $V$ is the potential, $\bar{J}_{i}$ is the flux of ionic species $i$ (number of particles per unit area), $D_{i}$ is the diffusion constant, $n_{i}$ is the concentration, and the constant $\alpha_{i}$ is defined as

$$
\alpha_{i}=\alpha / z_{i}
$$

with

$$
\alpha=R T / F
$$

where $z_{i}$ is the charge per ion, $R$ is the gas constant, $F$ is the Faraday constant, and $T$ is the temperature. The ionic concentrations and ionic currents must additionally satisfy the continuity equation:

$$
\bar{\nabla} \cdot \bar{J}_{i}+\partial n_{i} / \partial t=0
$$

The Nernst-Planck equation will be applied to a cylinder of diameter $d$. We assume that the longitudinal current and ionic concentrations are uniform across the transverse cross section of the cylinder and that the radial current is independent of angle around the axis of the cylinder. These assumptions reduce the problem of electrodiffusion to a one-dimensional problem along the axis of the cylinder. The constant-field approximation is made for the transverse currents passing through the cylinder (Goldman, 1943). The equations can be written in cylindrical coordinates and reduced to a single equation for the concentration as a function of the distance along the $z$ axis of a cylinder:

$$
\frac{\partial n_{i}}{\partial t}=D_{i} \frac{\partial^{2} n_{i}}{\partial z^{2}}+\frac{D_{i}}{\alpha_{i}} \frac{\partial}{\partial z}\left(n_{i} \frac{\partial V}{\partial z}\right)-\frac{4 P_{i} V}{\alpha_{i} d}\left[\frac{n_{i}^{o u t}-n_{i} e^{V / \alpha_{i}}}{1-e^{V / \alpha_{i}}}\right]
$$

where $P_{i}$ is the permeability of the membrane and $n_{i}{ }^{\text {out }}$ is the concentration of ionic species $i$ outside the membrane. The three terms on the right-hand side of this equation are, 
respectively, the contributions from pure diffusion, the potential gradient, and the membrane current. This equation must be supplemented by an additional constraint between the membrane potential and the ionic concentrations. We adopt the same capacitative model of the membrane used in the electrical conductance model:

$$
V(t)=V(0)+\sum_{i}\left[n_{i}(t)-n_{i}(0)\right] z_{i} F d / 4 c_{m}
$$

where $V(0)$ is the initial voltage, $n_{i}(0)$ are the initial ionic concentrations, and the membrane has capacitance $c_{m}$ per unit area.

If branches are allowed, then these equations must be solved on a tree rather than a line. At any jump in diameter, the continuity equation must be satisfied across the jump:

$$
\left.d_{1}^{2}\left(\frac{\partial n_{i}}{\partial z}+\frac{n_{i}}{\alpha_{i}} \frac{\partial V}{\partial z}\right)\right|_{1}=\left.d_{2}^{2}\left(\frac{\partial n_{i}}{\partial z}+\frac{n_{i}}{\alpha_{i}} \frac{\partial V}{\partial z}\right)\right|_{2}
$$

where the diameter of the process is $d_{1}$ on one side and $d_{2}$ on the other side. This implies that both the voltages and the ionic concentrations are continuous. However, by equation 6 , the voltage will not be continuous at a diameter jump if the ionic concentrations are continuous; hence, the voltage at the jump is set to the average of the voltages on either side.

The coupled differential equations were solved by converting them to finite difference equations and solving them by an explicit method. The solutions at diameter jumps were obtained by solving the coupled nonlinear algrebraic equations derived from the matching conditions, equation 7 . The calculation was performed for space and time steps of successively smaller size, and the values reported were ones for which further decrease to the step sizes made less than $2 \%$ difference to the solution.

\section{RELATIONSHIP BETWEEN THE ELECTRODIFFUSION MODEL AND THE ELECTRICAL CONDUCTANCE MODEL}

In large neurons, the internal and external ionic concentrations remain approximately constant during a transient excitation. The contribution of the diffusion of ions within the neuron then becomes negligible, and the longitudinal current is purely resistive. It can then be shown that

$$
\begin{aligned}
& 1 / R_{t}=\sum_{i}\left(1 / R_{i}\right) \\
& 1 / R_{i}=\left(F_{2} / R T\right) D_{i} n_{i} z_{i}
\end{aligned}
$$

where $R_{t}$ is the total resistivity of the cytoplasm and $R_{t}$ are the ionic resistivities for each species of ion. For the squid cytoplasm $\left(\left[\mathrm{K}^{+}\right]=400 \mathrm{mM},\left[\mathrm{Na}^{+}\right]=50 \mathrm{mM}\right)$ and for the $D_{i}$ given in Table I, the estimated resistivities are $R_{t}=29.7 \Omega \mathrm{cm}, R_{K}=33.4 \Omega$ $\mathrm{cm}$, and $R_{N a}=267 \Omega \mathrm{cm}$. There is a significant difference between the resistivities of the individual ionic species. 
TABLE I. Parameters for Electrodiffusion Model in Figs. 2, 3, and 4

\begin{tabular}{lll}
\hline Symbol & \multicolumn{1}{c}{ Value } & \multicolumn{1}{c}{ Parameter } \\
\hline$D_{K}$ & $1.96 \times 10^{-5} \mathrm{~cm}^{2} / \mathrm{sec}$ & Diffusion coefficient for $\mathrm{K}^{+}$ \\
$D_{N a}$ & $1.33 \times 10^{-5} \mathrm{~cm}^{2} / \mathrm{sec}$ & Diffusion coefficient for $\mathrm{Na}^{+}$ \\
$P_{K}$ & $3.64 \times 10^{-6} \mathrm{~cm}^{\mathrm{sec}}$ & Resting permeability of $\mathrm{K}^{+}$ \\
$P_{N a}$ & Resting permeability of $\mathrm{Na}^{+}$ \\
{$[\mathrm{K}]_{\text {in }}(0)$} & $6.07 \times 10^{-8} \mathrm{~cm} / \mathrm{sec}$ & Initial internal $\mathrm{K}^{+}$concentration \\
{$[\mathrm{Na}]_{\text {in }}(0)$} & $140 \mathrm{mM}$ & Initial $\mathrm{Na}^{+}$concentration \\
{$[\mathrm{K}]_{\text {out }}$} & $12 \mathrm{mM}$ & External $\mathrm{K}^{+}$concentration \\
{$[\mathrm{Na}]_{\text {out }}$} & $4 \mathrm{mM}$ & External $\mathrm{Na}^{+}$concentration \\
$P_{M}$ & $145 \mathrm{mM}$ & Maximum $\mathrm{Na}^{+}$permeability of spine \\
$t_{p}$ & $6.07 \times 10^{-3} \mathrm{~cm} / \mathrm{sec}$ & Time to reach peak permeability \\
$T$ & $0.25 \times 10^{-3} \mathrm{sec}$ & Temperature \\
$c_{m}$ & $20^{\circ} \mathrm{C}$ & Membrane capacitance per unit area \\
\hline
\end{tabular}

\section{ELECTRODIFFUSION MODEL OF A DENDRITIC SPINE}

Many vertebrate and invertebrate neurons receive synaptic inputs on spines (Coss and Perkel, 1985). Because of the small size of dendritic spines, postsynaptic potentials can be accompanied by significant changes in the internal ionic concentrations. In this section we simulate an excitatory postsynaptic potential on a spine using the electrodiffusion model and compare the results with the conventional electrical conductance model.

The morphology of the dendritic spine used in the simulations is shown in Fig. 1. The synaptic input was modeled by a transient change in the $\mathrm{Na}^{+}$permeability of the membrane

$$
P_{N a}=P_{M}\left(e t / t_{p}\right)^{4} e^{-4 t t_{p}}
$$

where $P_{M}$ is the maximum $\mathrm{Na}^{+}$permeability and $t_{p}$ is the time to reach peak (Kock and Poggio, 1983).

With the parameters of the model given in Table I, the resting potential was -78 $\mathrm{mV}$. The total surface area of the spine head was $0.65 \mu \mathrm{m}^{2}$. The membrane potential

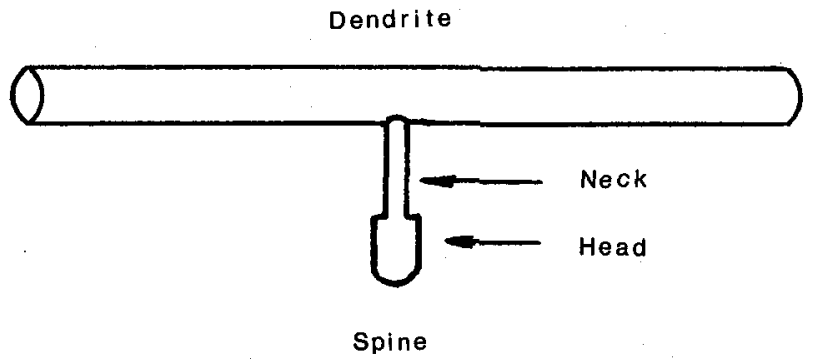

FIGURE 1. Geometry for the electrodiffusion model of a dendritic spine. The spine was in the center of a dendrite with a total length of $300 \mu \mathrm{m}$ and a diameter of $1 \mu \mathrm{m}$; the spine neck was $1 \mu \mathrm{m}$ long and $0.1 \mu \mathrm{m}$ in diameter; the spine head was $0.69 \mu \mathrm{m}$ long and $0.3 \mu \mathrm{m}$ in diameter. Sample points in the dendrite were $10 \mu \mathrm{m}$ apart, and the integration time step was $10^{-7} \mathrm{sec}$; in the spine head and neck the spacing was $0.173 \mu \mathrm{m}$ and $0.167 \mu \mathrm{m}$, respectively; and the time steps were $10^{-9} \mathrm{sec}$. The model had a total of 41 sample points: 31 in the dendrite, six in the spine neck, and four in the spine head. 
FIGURE 2. Excitatory postsynaptic potential modeled by electrodiffusion in a dendritic spine. The membrane potential relative to the resting potential is given as a function of time for the (1) middle of the spine head, (2) middle of the spine neck, (3) dendritic shaft at the base of spine, (4) dendrite $50 \mu \mathrm{m}$ from spine, and (5) dendrite $150 \mu \mathrm{m}$ from spine.

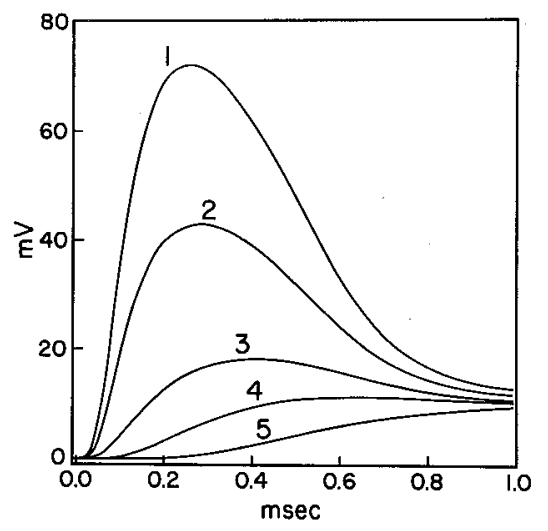

during the simulated excitatory postsynaptic potential is shown in Fig. 2, and the changes in the ionic concentrations of sodium and potassium are shown in Fig. 3. There is an increase in the sodium concentration inside the spine head of over threefold and a reduction in the concentration of potassium of $20 \%$. In Fig. 4, the maximum response is shown as a function of the maximum sodium permeability during the excitatory postsynaptic potential.

The parameters given in Table II for the electrical conductance model were chosen so that the resting and equilibrium potentials and the resting currents of the electrodiffusion model closely matched those in the electrical conductance model. The transient change in the membrane conductance of $\mathrm{Na}^{+}$at the spine head during the synaptic input was modeled by

$$
G_{N a}=G_{M}\left(e t / t_{p}\right)^{4} e^{-4 t / t_{p}}
$$

where $G_{M}$ is the maximum $\mathrm{Na}^{+}$conductance.

a

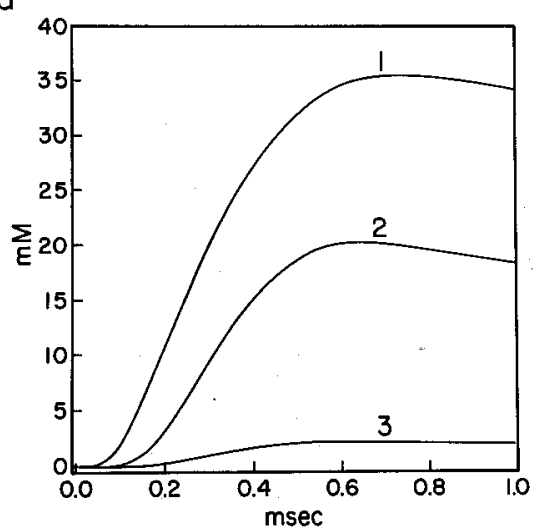

b

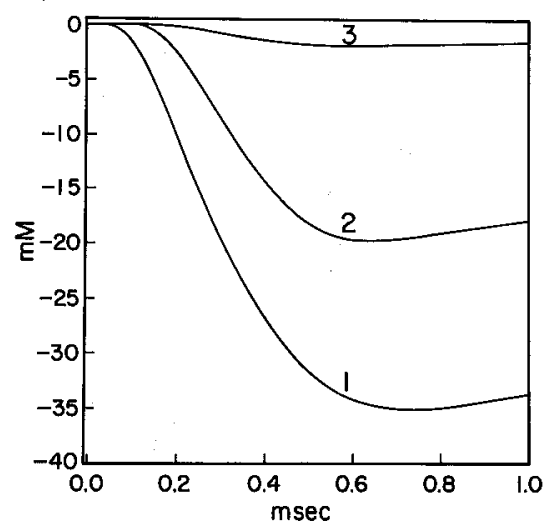

FIGURE 3. Ionic concentration changes for (a) $\mathrm{Na}^{+}$and (b) $\mathrm{K}^{+}$in a dendritic spine during an excitatory postsynaptic potential using the electrodiffusion model. Concentrations are given relative to the resting levels (see Table I) in the (1) middle of the spine head, (2) middle of the spine neck, and (3) dendritic shaft at the base of the spine. 


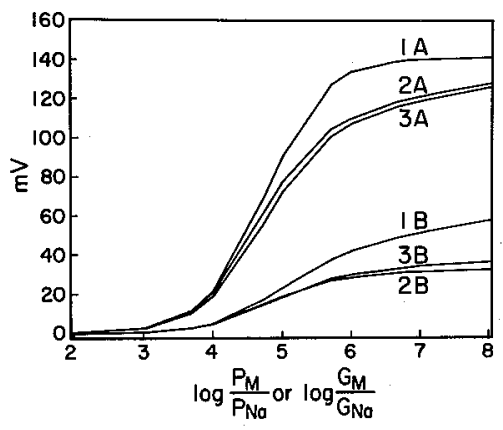

FIGURE 4. Maximum response during an excitatory postsynaptic potential for (1) the electrical conductance model and (2) the modified electrical conductance model as a function of the maximum conductance change and for (3) the electrodiffusion model as a function of the maximum permeability. The response functions for all three models are given at two locations: $(A)$ the spine head and $(B)$ the dendritic shaft at the base of the spine.

For small conductance changes, the two models predicted similar responses, as shown in Fig. 4. However, for large conductance changes, there were significant differences between the responses predicted by the electrical conductance model and the electrodiffusion model, especially at the base of the dendritic spine.

The saturation of the response in the electrical conductance model results from the approach of the membrane potential toward the sodium equilibrium potential. This saturation occurs at a lower membrane potential in the electrodiffusion model because of the increase in the internal sodium concentration and concomitant decrease of the sodium equilibrium potential.

The discrepancy between the two models can be reduced by using conductance changes in the electrical conductance model to match the membrane currents in the electrodiffusion model rather than the conductance changes given by equation 11 . However, this procedure requires a complete solution of the electrodiffusion equations first. An alternative modification of the electrical conductance model is presented in the next section that is computationally less demanding.

\section{MODIFICATIONS TO THE ELECTRICAL CONDUCTANCE MODEL}

In the conventional electrical conductance model, the ionic concentrations inside the neuron are constant during changes in the membrane potential. This assumption can be relaxed by making several changes to the formalism:

1. Calculate the concentration of each ionic species in each compartment from the ionic currents flowing between compartments.

TABLE II. Parameters for Electrical Conductance Model in Fig. 4

\begin{tabular}{lll}
\hline Symbol & \multicolumn{1}{c}{ Value } & \multicolumn{1}{c}{ Parameter } \\
\hline$g_{i K}$ & $5.56 \times 10^{-3} \mathrm{~S} / \mathrm{cm}$ & Cytoplasmic conductance of $\mathrm{K}^{+}$ \\
$g_{i N a}$ & $5.56 \times 10^{-3} \mathrm{~S} / \mathrm{cm}$ & Cytoplasmic conductance of $\mathrm{Na}^{+}$ \\
$g_{m K}$ & $2.31 \times 10^{-4} \mathrm{~S} / \mathrm{cm}^{2}$ & Resting membrane conductance of $\mathrm{K}^{+}$ \\
$g_{m N a}$ & $1.94 \times 10^{-5} \mathrm{~S} / \mathrm{cm}^{2}$ & Resting membrane conductance of $\mathrm{Na}^{+}$ \\
$E_{K}$ & $-89.8 \mathrm{mV}$ & $\mathrm{K}^{+}$equilibrium potential \\
$E_{N a}$ & $62.9 \mathrm{mV}$ & $\mathrm{Na}^{+}$equilibrium potential \\
$G_{M}$ & $1.26 \times 10^{-8} \mathrm{~S}$ & Maximum sodium conductance of spine \\
$t_{p}$ & $0.25 \times 10^{-3} \mathrm{sec}$ & Time to reach peak conductance \\
$c_{m}$ & $2 \mu \mathrm{F} / \mathrm{cm}^{2}$ & Membrane capacitance per unit area \\
\hline
\end{tabular}


2. Compute the new equilibrium potentials during each time step and update the membrane batteries.

3. Replace the longitudinal resistance between compartments with parallel conductances in series with batteries and treat them in the same way as membrane conductances.

This modified electrical conductance model applied to the dendritic spine model in Fig. 1 gave qualitatively similar results for the changes in ionic concentrations compared with the electrodiffusion model. Without the above modifications, the predicted ionic concentration changes were markedly in error, in some cases having the wrong sign. The modified electrical conductance model also made predictions for the maximum responses that were qualitatively similar to those of the electrodiffusion model, with quantitative discrepancies of less than $10 \%$ over the entire range of conductance changes, as shown in Fig. 4.

\section{DISCUSSION}

In most circumstances, the electrical conductance model of electrical conduction in neurons gives accurate predictions for membrane potentials during transient electrical events. In this chapter we have developed an electrodiffusion model of electrical conduction for thin processes that reduces to the electrical conductance model for processes with large diameters.

This one-dimensional electrodiffusion model was used to study the changes in concentration of ions in dendritic spines during excitatory postsynaptic potentials. During a conductance change for $\mathrm{Na}^{+}$at the distal tip of a spine, the concentration of sodium can transiently increase by threefold, and the potassium concentration can decrease by $20 \%$. Thus, significant errors can be made in estimating the membrane potential and concentration changes with the electrical conductance model if the effects of diffusion are not taken into account. We suggest a modification of the electrical conductance model to minimize these errors.

In a later paper we will extend the present model by including membrane pumps, buffers, and other ions, such as $\mathrm{Ca}^{2+}$, that may also be important (Simon and Llinas, 1985; Fogelson and Zucker, 1985). For some problems it may be necessary to include spatially inhomogeneous diffusion within neurons, which would require the solution of the Nernst-Planck equation in three dimensions.

ACKNOWLEDGMENTS. We are grateful to Drs. Julian Jack, Wilfred Rall, and John Rinzel for helpful comments on earlier versions of this chapter.

\section{REFERENCES}

Coss, R. G., and Perkel, D. H., 1985, The function of dendritic spines, Behav. Neural Biol. 44:151-185.

Fogelson, A. L., and Zucker, R. S., 1985, Presynaptic calcium diffusion from various arrays of single channels, Biophys. J. 48:1003-1017.

Goldman, D. E., 1943, Potential, impedance and rectification in membranes, J. Gen. Physiol. 27:37-60. 
Hodgkin, A. L., and Huxley, A. F. 1952, Currents carried by sodium and potassium ions through the membrane of the giant axon of Loligo, J. Physiol. (Lond.) 116:449-472.

Jack, J. J. B., Noble, D., and Tsien, R. W., 1975, Electrical Current Flow in Excitable Cells, Oxford University Press, Oxford.

Koch, C., and Poggio, T., 1983, A theoretical analysis of electrical properties of spines, Proc. R. Soc. Lond. [Biol.] 218:455-477.

Koch, C., Poggio, T., and Torre, V., 1983, Nonlinear interaction in a dendritic tree: Location, timing, and role in information processing, Proc. Natl. Acad. Sci. U.S.A. 80:2799-2802.

Perkel, D. H., and Perkel, D. J., 1985, Dendritic spines: Role of active membrane modulating synaptic efficacy, Brain Res. 325:331-335.

Rall, W., 1977, Core conductor theory and cable properties of neurons, in: Handbook of Physiology: The Nervous System (E. R. Kandel, ed.), American Physiological Society, Bethesda, pp. 39-97.

Rall, W., 1978, Dendritic spines and synaptic potency, in: Studies in Neurophysiology (R. Porter, ed.), Cambridge University Press, Cambridge, pp. 203-209.

Rall, W., and Segev, I., 1987, Functional possibilities for synapses on dendrites and dendritic spines, in: New Insights into Synaptic Function (G. M. Edelman, W. F. Gall, and W. M. Cowan, eds.), John Wiley \& Sons, New York (in press).

Shepherd, G. M., Brayton, R. K., Miller, J. P., Segev, I., Rinzel, J., and Rall, W., 1985, Signal enhancement in distal cortical dendrites by means of interactions between active dendritic spines, Proc. Natl. Acad. Sci. U.S.A. 82:2192-2195.

Simon, S. M., and Llinas, R. R., 1985, Compartmentalization of the submembrane calcium activity during calcium influx and its significance in transmitter release, Biophys. J. 48:485-498. 